\title{
Polymer constraint effect for electrothermal bimorph microactuators
}

\author{
T. Chu Duc ${ }^{\text {a) }}$ \\ Laboratory of Electronic Components, Technology and Materials, DIMES, Delft University of Technology, \\ Feldmannweg 17, 2628 CT Delft, The Netherlands
}

G. K. Lau

Department of Precision and Microsystems Engineering, 3ME, Delft University of Technology, Delft 2628 $C D$, The Netherlands

P. M. Sarro

Laboratory of Electronic Components, Technology and Materials, DIMES, Delft University of Technology, Feldmannweg 17, 2628 CT Delft, The Netherlands

(Received 16 July 2007; accepted 14 August 2007; published online 4 September 2007)

\begin{abstract}
The authors report on the analysis of the polymer constraint effect and its use for a micromachined electrothermal bimorph actuator. The actuated displacement is enhanced due to the polymer constraint effect. Both the thermal expansion and apparent Young's modulus of the constrained polymer blocks are significantly improved, compared with the no constraint case. The calculation that agrees well with experimental results provides the means to optimize the design of the constrained polymer stack electrothermal microactuator. (c) 2007 American Institute of Physics.
\end{abstract}

[DOI: $10.1063 / 1.2779929]$

Thermal expansion of polymer is currently widely used in microactuators to produce large motion at low temperature. In most of the polymeric electrothermal actuators reported in literature, a thin metal layer deposited on the top surface of the polymer structures is employed as heater. ${ }^{1,2}$ Hence, the heat transfer interface between the heater and the polymer is rather limited. Since the polymer layers have low heat conduction rate, the actuator dimensions need to be small enough to get a good vertical temperature profile in the structure. Consequently, the resulting motion is quite limited. Moreover, the unintended vertical movement couples and interferes with the desired lateral movement of the structures.

In this letter, we report on a silicon-polymer electrothermal bimorph actuator with a large motion range. This actuator has a silicon comb finger structure with a metal heater on top. The gaps between comb fingers are filled with polymer [see Fig. 1(a)]. When the heater is activated, the generated heat is efficiently transferred to the surrounding polymer through the deep silicon comb finger structures that have a large heat transfer interface area with the polymer layers. The polymer layers expand along the lateral direction causing bending displacement of the bimorph cantilever actuator. Moreover, the thermal expansion of this actuator is enhanced due to the constraint effect of polymer layers between rigid silicon plates. ${ }^{3}$

A calculation of compressed displacement of a constrained rubber between two rigid surfaces based on the hydrostatic pressure is reported in Ref. 4. The hydrostatic pressure theory is expanded here by taking into account the thermal expansion of constrained polymer as a function of the ratio between the dimensions of the rigid plate and the thickness of the polymer block. Finally, the thermal displacement of the actuator is then calculated using bimorph bending theory.

It is assumed that the plates have infinite length, the thickness of the polymer block $h$ is much smaller than the

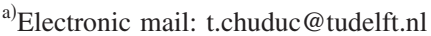

width $w$ of the plate $(h \ll w)$, and the polymer is incompressible (Poisson's ratio $\nu_{S}=0.5$ ). When the temperature of the structure changes by $\Delta T$ degree, the volume thermal expansion of a polymer block is given by ${ }^{5}$

$$
\Delta V=3 \alpha_{T} \Delta T V,
$$

where $V$ is the initial volume of the polymer block and $\alpha_{T}$ is the thermal expansion coefficient (CTE) of the polymer.

In the case of no constraint, the volume of the polymer block expands in three dimensions, as indicated by the dashed line in Fig. 2. When the polymer block is constrained by two rigid parallel plates, the thermal expansion along the

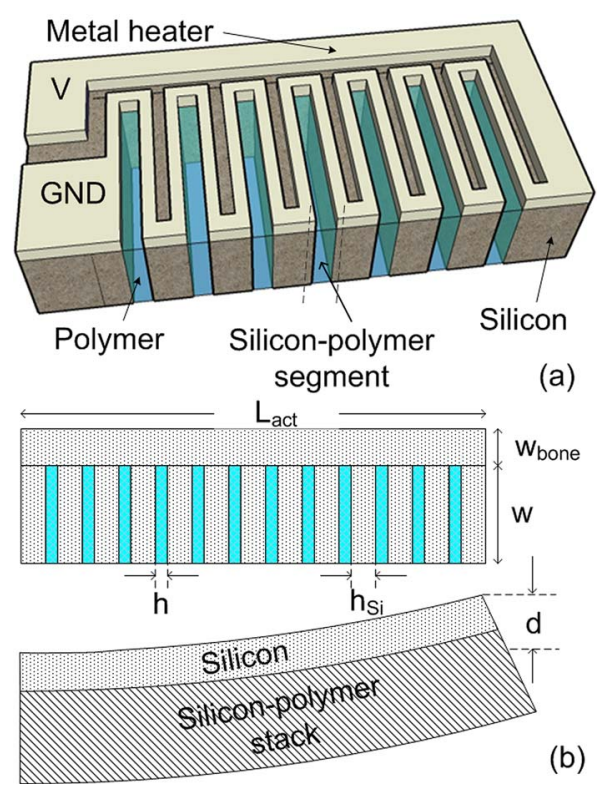

FIG. 1. (Color online) Sketch of the proposed silicon-polymer electrothermal actuator: (a) device consists of silicon comb structure with a thin heater on the top and the filled polymer in between the comb fingers; (b) the actuator is simply considered as the bimorph structure of silicon bone and silicon-polymer lateral stack. 


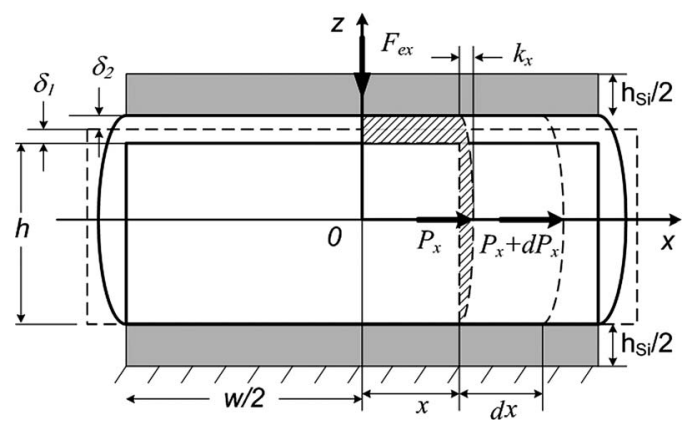

FIG. 2. Cross section of a constrained polymer block between two rigid silicon plates.

$z$ axis consists of two parts. The first one is the regular thermal expansion $\delta_{1}$ that is the same as for the no constraint one and is given by

$$
\delta_{1}=h \alpha_{T} \Delta T \text {. }
$$

The second part is the displacement $\delta_{2}$, which is the subsequent shear displacement necessary to restore points in the planes of the rigid surfaces to their original position in these planes. The corresponding strain $e$ is given by $e$ $=\delta_{2} / h$.

There are two corresponding internal forces $F_{1}$ and $F_{2}$ applied to the rigid surfaces. The force $F_{1}$ (unit in $\mathrm{N} / \mathrm{m}$ ), corresponding to the pure homogeneous strain $e$, on the surface of the rigid plates per unit length of the block, is given by $^{4}$

$$
F_{1}=f_{c} w E e=\frac{4}{3} w E e,
$$

where $f_{c}=4 / 3$ is a numerical factor representing the restrictive effect of the bonded surface, $w$ is the width of the block, and $E$ is Young's modulus of the polymer.

When $w \gg h$, the horizontal planes remain plane during the deformation and the vertical planes consequently take up the parabolic forms, as represented in Fig. 2. ${ }^{4}$ The expanded volume $\Delta V$ is the shadowed area between the central plane and the plane far from the center at a distance $x$. Compared with the expansion volume from Eq. (1), the maximum outward displacement $k_{x}$ of the vertical plane at $x$ is given by

$$
k_{x}=\frac{3}{2}\left(2 \alpha_{T} \Delta T-e\right) x .
$$

Displacement of a section of width $d x$ may be maintained by an excess hydrostatic pressure $d P_{x}$ given by ${ }^{4}$

$$
d P_{x}=-\frac{8}{3} \frac{E k_{x}}{h^{2}} d x
$$

The pressure $P_{x}$ denotes the hydrostatic pressure at $x$ and is zero at the boundary $(x= \pm w / 2)$ and is obtained by integration,

$$
P_{x}=\int_{w / 2}^{x} d P_{x}=\frac{2 E\left(2 \alpha_{T} \Delta T-e\right)}{h^{2}}\left(\frac{w^{2}}{4}-x^{2}\right) .
$$

The pressure $P_{x}$ is considered to act on the rigid plates also. The corresponding force $F_{2}$ is therefore given by

$$
F_{2}=\frac{1}{3} \frac{E\left(2 \alpha_{T} \Delta T-e\right)}{h^{2}} w^{3} .
$$

The total force applied to the unit length of the rigid surface at steady state is $\mathbf{F}=\mathbf{F}_{1}+\mathbf{F}_{2}+\mathbf{F}_{\text {ex }}=0$, where $F_{\text {ex }}$ (unit Downloaded 10 Aug 2010 to 131.180.130.114. Redistribution subje in $\mathrm{N} / \mathrm{m}$ ) is the external applied force per unit length of the structure. Combining Eqs. (3) and (7), the hydrostatic strain of the thermal expansion of a constrained polymer block is given by

$$
e=\frac{2 \alpha_{T} \Delta T w^{2}}{4 h^{2}+w^{2}}-\frac{3 h^{2} F_{\mathrm{ex}}}{w E\left(4 h^{2}+w^{2}\right)} .
$$

The total thermal expansion $\delta$ of the constrained polymer block is the sum of the regular expansion $\delta_{1}$ and the hydrostatic displacement $\delta_{2}$ and is given by

$$
\delta=h \alpha_{T} \Delta T+\frac{2 \alpha_{T} \Delta T w^{2} h}{4 h^{2}+w^{2}}-\frac{3 h^{3}}{E w\left(4 h^{2}+w^{2}\right)} F_{\mathrm{ex}} .
$$

The apparent CTE of the constrained polymer between two rigid plates $\alpha_{\text {app }}$ and consequently the CTE of the silicon-polymer lateral stack $\alpha_{\text {stack }}$ can be calculated from Eq. (9) when the external applied force is zero and is given by

$$
\begin{aligned}
& \alpha_{\text {app }}=\alpha_{T}\left(1+\frac{2 w^{2}}{4 h^{2}+w^{2}}\right) \simeq 3 \alpha_{T}, \\
& \alpha_{\text {stack }}=\frac{\alpha_{\mathrm{app}} h+\alpha_{\mathrm{Si}} h_{\mathrm{Si}}}{h+h_{\mathrm{Si}}} \simeq \alpha_{\mathrm{app}} \frac{h}{h+h_{\mathrm{Si}}},
\end{aligned}
$$

where $\alpha_{\mathrm{Si}}$ is CTE of silicon and $h_{\mathrm{Si}}$ is thickness of the silicon plates. The approximation of $\alpha_{\text {app }} \simeq 3 \alpha_{T}$ is valid when $w$ $\gg h$; the thermal expansion of the constrained polymer layer reaches the stable value when the $w / h$ ratio is about 10 . The $\alpha_{\text {stack }}$ approximation is valid due to the small thermal expansion of silicon.

Beside the expansion, the apparent Young modulus of the constrained polymer $E_{\mathrm{app}}$ and the modulus of the siliconpolymer stack $E_{\text {stack }}$ are also important parameters of the actuator. ${ }^{6}$ They are given by

$$
\begin{aligned}
& E_{\text {app }}=E \frac{1}{3}\left(4+(w / h)^{2}\right), \\
& E_{\text {stack }}=\frac{E_{\mathrm{Si}} E_{\mathrm{app}}\left(h_{\mathrm{Si}}+h\right)}{h_{\mathrm{Si}} E_{\mathrm{app}}+h E_{\mathrm{Si}}},
\end{aligned}
$$

where $E_{\mathrm{Si}}$ is Young's modulus of silicon. Young's modulus of the silicon-polymer stack structure increases with the second-order of the $w / h$ ratio.

As shown in Fig. 1(b), the silicon-polymer electrothermal actuator proposed here is simply considered as the bimorph structure consisting of two layers: silicon bone and silicon-polymer lateral stack. The thermal displacement $d$ at the free end of the bimorph structure cantilever can be calculated based on the Timoshenko calculation ${ }^{7}$ and is given by

$$
d=\frac{3 L_{\mathrm{act}}^{2}\left(\alpha_{\text {stack }}-\alpha_{\mathrm{Si}}\right)(1+m)^{2} \Delta T}{\left(w+w_{\text {bone }}\right)\left(3(1+m)^{2}+(1+m n)\left(m^{2}+\frac{1}{m n}\right)\right)},
$$

where $L_{\text {act }}$ is the length of actuator, $w_{\text {bone }}$ is the width of silicon bone, $w$ is the width of silicon and polymer plates, $n=E_{\mathrm{Si}} / E_{\text {stack }}$, and $m=w_{\text {bone }} / w$.

As polymer, we have selected SU8 2002 (Microchem Inc.). The related parameters of SU8 are assumed to be temperature independent. The polymer CTE is $150.7 \pm 12$ and to AIP license or copyright; see http://apl.aip.org/apl/copyright.jsp 


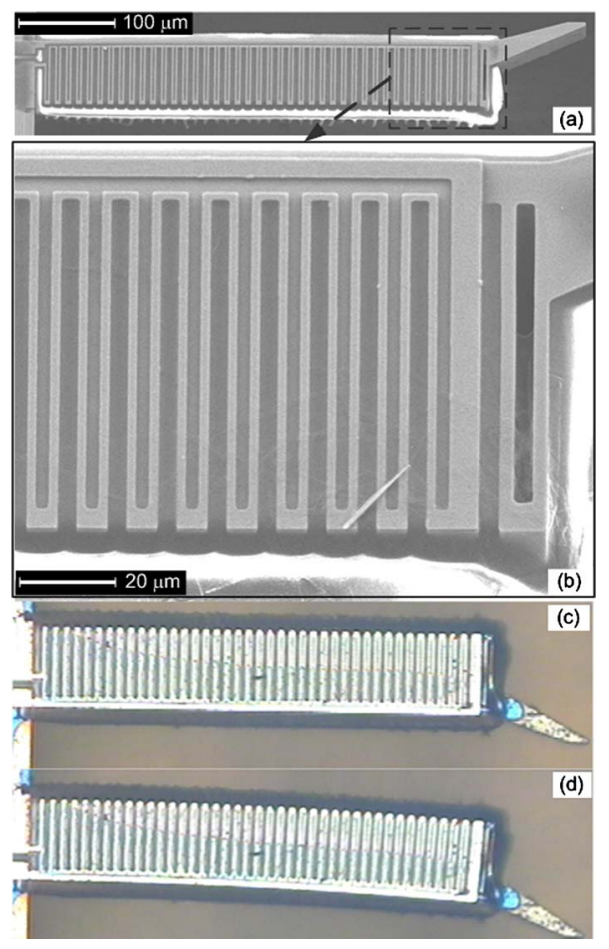

FIG. 3. (Color online) Fabricated device: (a) the entire structure; (b) close view of silicon-polymer stack; [(c) and (d)] pictures of the initial and actuated states (the bimorph bends up to $14 \mu \mathrm{m}$ when it is heated).

$2.6 \mathrm{ppm} / \mathrm{K}$ for SU8 polymer and silicon, respectively. ${ }^{8,9}$ Young's modulus of the SU8 polymer is $3.2 \mathrm{GPa}$, which is 53 times softer than that of silicon. ${ }^{8,10}$

To experiment the validity of the calculation, the proposed design is fabricated using the process described elsewhere. ${ }^{1 P}$ The discussed actuator is $85 \mu \mathrm{m}$ wide and $30 \mu \mathrm{m}$ thick. It consists of 41 polymer-silicon fingers. Silicon fingers are $75 \mu \mathrm{m}$ wide and $6 \mu \mathrm{m}$ thick with $600 \mathrm{~nm}$ thick aluminum heater on the top. There are two configurations of SU8 polymer layer with $3 \mu \mathrm{m}(w / h=25)$ and $5 \mu \mathrm{m}$ thickness $(w / h=15)$ for the first and second structures, respectively. The silicon bone width is $10 \mu \mathrm{m}$. The vertical silicon combs and frame structure are fabricated using deep reactive ion etching of silicon. The SU8 2002 polymer is then filled among the trenches. Finally, the structure is released from the substrate by a combination of wet and dry etching of the silicon substrate. Figure 3 shows the scanning electron microscopy images of the fabricated device and the bent structure when actuated.

The structure is measured by using the Cascade probe station (Cascade Microtech, Inc.) with a built-in microscope to measure the displacement. A dc voltage is applied on the metal heater by using HP4155A semiconductor parameter analyzer (Agilent Technologies, Inc.). The voltage is ramped from 0 to $2.5 \mathrm{~V}$. The displacement is monitored through the charge coupled device camera on the top of the probe station. The displacement of the actuator at any actuating voltage is then obtained by enlarging the picture and comparing with the initial picture. With this method, the inaccuracy is given by the single pixel size that is $0.26 \times 0.26 \mu \mathrm{m}^{2}$. However, the external mechanical vibration causes a blur on the static picture which mainly determines the inaccuracy of the measurement. The total estimated measurement error is about

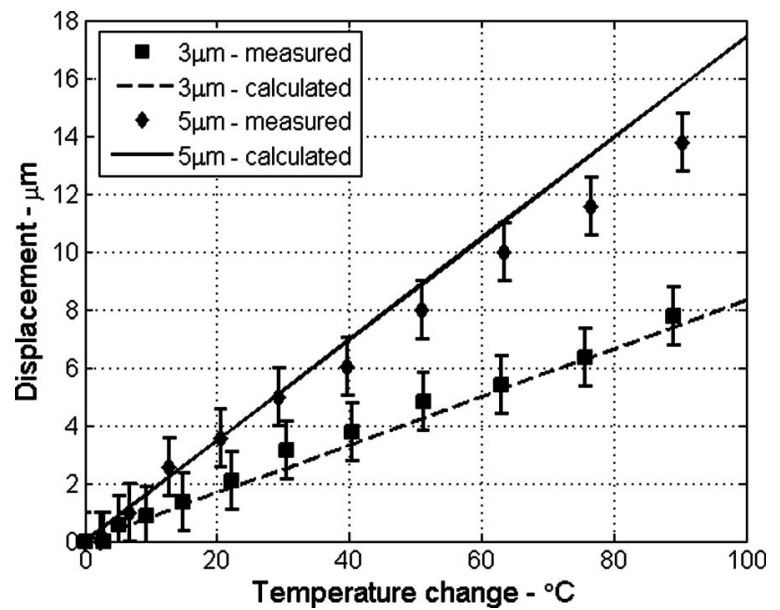

FIG. 4. Displacement of the silicon-polymer electrothermal actuator vs the operating temperature for 3 and $5 \mu \mathrm{m}$ thick SU8 layer devices.

$\pm 1.0 \mu \mathrm{m}$. The average increasing temperature in the electrothermal actuator can be estimated by monitoring the change of the resistance of the aluminum heater.

The measured displacements reach 8 and $14 \mu \mathrm{m}$ at $2.5 \mathrm{~V}$ applied voltage for 3 and $5 \mu \mathrm{m}$ thick polymer layers, respectively. The power consumption, calculated through the applied voltage and corresponding current, is $\sim 24 \mathrm{~mW}$ at $2.5 \mathrm{~V}$ for both devices. Figure 4 shows the calculated and measured displacements of the proposed devices versus the operating temperature in the actuators. The calculated thermal displacements meet the experimental results within $2 \%$ and $5 \%$ for the 3 and $5 \mu \mathrm{m}$ thick polymer layers, respectively.

In summary, we have investigated a polymeric electrothermal microactuator with an enhanced displacement due to the polymer constraint effect. The apparent CTE of the constrained polymer block is three times larger than the no constraint one when $w \gg h$. Our theoretical results are in agreement with the measurement. This structure has great potential for in-plane motion actuators for micro electromechanical system devices with the additional advantage of using low applied voltage and low operating temperature.

This work has been partly supported by the Vietnamese Ministry of Education and Training. The authors would like to acknowledge the DIMES-IC Processing group for technical support. The many discussions with J. Wei, M. Saadaoui, and J. F. Creemer are greatly appreciated.

${ }^{1}$ N. Chronis and L. P. Lee, J. Microelectromech. Syst. 14, 857 (2005).

${ }^{2}$ N. T. Nguyen, S. S. Ho, and C. L. N. Low, J. Micromech. Microeng. 14, 967 (2004).

${ }^{3}$ G. K. Lau, J. F. L. Goosen, F. van Keulen, T. Chu Duc, and P. M. Sarro, Appl. Phys. Lett. 90, 214103 (2007).

${ }^{4}$ A. N. Gent and E. A. Meinecke, Polym. Eng. Sci. 10, 48 (1970).

${ }^{5}$ S. D. Senturia, Microsystem Design (Kluwer Academic, Dordrecht, 2001), pp. $183-200$.

${ }^{6}$ A. N. Gent and P. B. Lindley, Proc. Inst. Mech. Eng. 173, 111 (1959).

${ }^{7}$ S. Timoshenko, J. Opt. Soc. Am. 11, 233 (1925)

${ }^{8}$ R. Feng and R. J. Farris, J. Micromech. Microeng. 13, 80 (2003).

${ }^{9}$ Y. Okada and Y. Tokumaru, J. Appl. Phys. 56, 314 (1984).

${ }^{10}$ J. J. Wortman and R. A. Evans, J. Appl. Phys. 36, 153 (1965).

${ }^{11}$ T. Chu Duc, G. K. Lau, J. Wei, and P. M. Sarro, Proceedings of Fifth IEEE Conference on Sensors, Daegu, Korea, 22-26 October 2006 (IEEE, New York, 2007), pp. 662-665. 